\title{
Generalização de um truque matemático
}

\author{
Rogério César dos Santos(1) \\ Wescley Well Vicente Bezerra(D)
}

\author{
Paulo Eduardo de Brito(i) \\ Carlos Derli Almeida Cornélio(i)
}

\section{Resumo}

O intuito do presente artigo é generalizar o truque matemático apresentado por Santos e Gontijo (2018)[1]. Na mágica, o adivinhador disponibiliza para um participante voluntário T, 7 bolinhas iguais para que o participante escolha uma certa quantidade dessas bolinhas e as distribua em suas duas mãos, tudo secretamente. Depois, informa ao adivinhador o resultado da soma $\mathrm{S}=$ $2 \mathrm{x}+3 \mathrm{y}$, onde $\mathrm{x}$ é a menor quantidade de bolinhas numa mão e y a maior, para que o mesmo descubra quantas bolinhas o participante escolheu e como elas foram distribuídas em suas mãos. Os coeficientes 2 e 3 são chamados de multiplicadores. Nesse artigo será demonstrado o funcionamento da mágica para o caso mais geral de qualquer quantidade total $\mathrm{T}$ de bolinhas, e também quais devam ser os multiplicadores apropriados. Para a prova, serão utilizados resultados básicos da teoria de divisibilidade nos inteiros.

Palavras-chave: Lúdico; Mágica Matemática; Divisibilidade.

\begin{abstract}
The purpose of this paper is to generalize the mathematical trick presented by Santos and Gontijo (2018)[1]. In this magic, the diviner makes it available to a volunteer participant T, 7 equal balls for the participant to choose a certain amount of these balls and distribute them in his two hands, all secretly. Then, he tells the diviner the result of the sum $S=2 x+3 y$, where $x$ is the smallest number of balls in one hand and $y$ is the largest, to find out how many balls the participant chose and how they were distributed in their hands. The coefficients 2 and 3 are called multipliers. This article will demonstrate how the magic works for the most general case of any total number of $\mathrm{T}$ balls, and also what the appropriate multipliers should be. For the proof, basic results of the theory of divisibility in integers will be used.
\end{abstract}

Keywords: Ludic; Mathematical Magic; Divisibility.

\section{Introdução}

Os truques matemáticos em sala de aula podem ser utilizados como uma importante atividade para motivar e prender a atenção dos alunos para que, num segundo momento, o professor possa trabalhar os conteúdos matemáticos presentes nessas mágicas. O artigo "Uma Mágica Desafiadora", de Santos e Gontijo (2018), traz uma instigante atividade lúdica, na qual podem ser trabalhados os conceitos de equação do primeiro grau, e sistemas dois por dois.

O enunciado da mágica presente no referido artigo é descrito a seguir: 
1) Peça para que um aluno voluntário segure 7 bolinhas (ou qualquer objeto pequeno como tampas ou moedas) e escolha secretamente um número w entre 3 e 7 , e, também secretamente, distribua essa quantidade de bolinhas nas mãos (nenhuma mão pode ficar vazia). Suponha, por exemplo, que ele tenha escolhido 4 bolinhas, distribuindo 1 numa mão e 3 na outra. Nenhum desses dados será informado a você.

2) Peça que ele mentalmente dobre a mão de menor quantidade e triplique a mão de maior quantidade e informe a soma $\mathrm{z}$ dos resultados. Se a distribuição das bolinhas tiver sido igual nas duas mãos, continue normalmente. No nosso exemplo, a soma z será $2 \cdot 1+3 \cdot 3=11$.

3) Você já pode adivinhar quantas bolinhas ao todo ele escolheu inicialmente: $\mathrm{w}=4$, e também como elas foram distribuidas: 1 e 3. Como? [1]

Neste trabalho, que objetiva generalizar tal truque, o total de bolinhas será denotado por T $(\mathrm{T}=7$ no referido artigo), os multiplicadores por u e v ( $\mathrm{u}=2 \mathrm{e} \mathrm{v}=3$ no artigo citado), a menor quantidade distribuída numa mão por x e a maior por y, e a soma ux + vy por S.

Vamos dividir nossa tarefa em três partes: primeiro, mostrar a unicidade da soma S para qualquer quantidade total $\mathrm{T}>0$ de bolinhas, desde que se escolham corretamente os multiplicadores $\mathrm{u}$ e v; segundo, mostrar como é possível descobrir as quantidades de bolinhas em cada mão e, por fim, será dado um exemplo de como proceder à realização do truque em uma situação particular.

\section{Unicidade da soma informada $S$ : prova algébrica}

Antes de mais nada, pensamos em quais seriam os multiplicadores adequados que garantem a unicidade da soma S. Por tentativa e erro, dado T, percebemos que basta os multiplicadores satisfazerem dois critérios: devem ser coprimos e também maiores ou iguais ao quociente da divisão de $\mathrm{T}$ por 2. O menor multiplicador multiplica a menor quantidade de bolinhas em uma mão e o maior multiplica a maior quantidade na outra mão. Nessas condições testamos computacionalmente para centenas de possibilidades, e verificamos a unicidade da soma S em todas elas. Assim, fomos levados a formular a proposição seguinte.

Considere inicialmente que o participante 1 escolha $\mathrm{Q}_{1}=\mathrm{x}_{1}+\mathrm{y}_{1}$ de um conjunto $\mathrm{T}$ de bolinhas tal que $0 \leq \mathrm{x}_{1} \leq \mathrm{y}_{1}$, e que um participante 2 escolha $\mathrm{Q}_{2}=\mathrm{x}_{2}+\mathrm{y}_{2}$ de um outro conjunto, também de $\mathrm{T}$ bolinhas, em que $0 \leq \mathrm{x}_{2} \leq \mathrm{y}_{2}$.

Teorema 1. Dado $\mathrm{T} \geq 0$, consideremos $\mathrm{N} \geq 0$ o quociente da divisão de $\mathrm{T}$ por 2 e $\mathrm{v}>\mathrm{u} \geq \mathrm{N}$, sendo $\mathrm{u} e \mathrm{v}$ coprimos maiores do que zero, os multiplicadores. Tomemos $\left(\mathrm{x}_{1}, \mathrm{y}_{1}\right) \neq\left(\mathrm{x}_{2}, \mathrm{y}_{2}\right)$, com $\mathrm{x}_{1} \leq \mathrm{y}_{1}$ e $\mathrm{x}_{2} \leq \mathrm{y}_{2}$ naturais tais que $\mathrm{Q}_{\mathrm{i}}=\mathrm{x}_{\mathrm{i}}+\mathrm{y}_{\mathrm{i}} \leq \mathrm{T}, \mathrm{i}=1,2$. Definindo

$$
\mathrm{S}_{\mathrm{i}}=\mathrm{ux}_{\mathrm{i}}+\mathrm{vy}_{\mathrm{i}} \quad 8 \mathrm{i}=1,2,
$$

então garante-se que

$\mathrm{S}_{1} \neq \mathrm{S}_{2}$. 
Demonstração. Suponhamos por contradição que existam inteiros T, N, u, v, x $1, \mathrm{y}_{1}, \mathrm{x}_{2}$ e $\mathrm{y}_{2}$ que cumprem as hipóteses da proposição e sejam tais que

$$
\mathrm{S}_{1}=\mathrm{ux}_{1}+\mathrm{vy}_{1}=\mathrm{S}_{2}=\mathrm{ux}_{2}+\mathrm{vy}_{2}
$$

Chegaremos em uma contradição, ao final das passagens seguintes. Da igualdade acima, temos:

$$
\mathrm{v}\left(\mathrm{y}_{1}-\mathrm{y}_{2}\right)=\mathrm{u}\left(\mathrm{x}_{2}-\mathrm{x}_{1}\right)
$$

Se $\mathrm{x}_{1}=\mathrm{x}_{2}$ então $\mathrm{y}_{1}=\mathrm{y}_{2}$, contrariando uma hipótese da proposição. Portanto, $\mathrm{x}_{1} \neq \mathrm{x}_{2} \mathrm{e}$, como $\mathrm{v}>\mathrm{u}>0$, tem-se que $\mathrm{y}_{1} \neq \mathrm{y}_{2}$.

Como v e u são coprimos, conclui-se, por (1) e da Teoria dos Números, que v deve ser divisor de $\mathrm{x}_{2}-\mathrm{x}_{1}$, ou seja, existe a inteiro não nulo tal que

$$
\mathrm{x}_{2}-\mathrm{x}_{1}=\mathrm{av}
$$

O inteiro não nulo a pode ser positivo ou negativo nesta equação.

Caso 1 Suponha inicialmente que a $>0$. O caso a $<0$ será tratado à parte. De (1),

$$
\begin{gathered}
\mathrm{v}\left(\mathrm{y}_{1}-\mathrm{y}_{2}\right)=\text { uav } \\
\mathrm{y}_{1}-\mathrm{y}_{2}=\mathrm{ua} .
\end{gathered}
$$

De $\mathrm{a} \geq 1$ e somando $\mathrm{x}_{2}-\mathrm{x}_{1}=$ av com $\mathrm{y}_{1}-\mathrm{y}_{2}=\mathrm{ua}$, temos

$$
\left(\mathrm{x}_{2}-\mathrm{x}_{1}\right)+\left(\mathrm{y}_{1}-\mathrm{y}_{2}\right)=\mathrm{av}+\mathrm{ua} \geq \mathrm{v}+\mathrm{u} \geq \mathrm{v}+\mathrm{N}>\mathrm{N}+\mathrm{N}=2 \mathrm{~N},
$$

de onde decorre que

$$
\mathrm{x}_{2}-\mathrm{x}_{1}+\mathrm{y}_{1}-\mathrm{y}_{2} \geq 2 \mathrm{~N}+1
$$

Bem, sabemos que $\mathrm{T}=2 \mathrm{~N}+1$ ou $\mathrm{T}=2 \mathrm{~N}$, conforme $\mathrm{T}$ seja ímpar ou par, respectivamente. De toda forma, é certo que $\mathrm{T} \leq 2 \mathrm{~N}+1$.

Então, como por hipótese $\mathrm{x}_{2}-\mathrm{y}_{2} \leq 0$, temos:

$$
\mathrm{x}_{2}-\mathrm{x}_{1}+\mathrm{y}_{1}-\mathrm{y}_{2} \leq \mathrm{y}_{1}-\mathrm{x}_{1} \leq \mathrm{y}_{1} \leq \mathrm{T} \leq 2 \mathrm{~N}+1
$$

Logo, de (2) e (3),

$$
\mathrm{x}_{2}-\mathrm{x}_{1}+\mathrm{y}_{1}-\mathrm{y}_{2}=2 \mathrm{~N}+1
$$

e as desigualdades em (3) na verdade são igualdades. Assim, $\mathrm{T}=2 \mathrm{~N}+1$ é ímpar! As igualdades em (3) ainda implicam que $\mathrm{y}_{1}-\mathrm{x}_{1}=\mathrm{y}_{1}=2 \mathrm{~N}+1$, o que só é possível se $\mathrm{x}_{1}=0$. Desta forma, de (4), $\mathrm{x}_{2}-0+2 \mathrm{~N}+1-\mathrm{y}_{2}=2 \mathrm{~N}+1$, e daí $\mathrm{x}_{2}=\mathrm{y}_{2}$. Logo, av $=\mathrm{x}_{2}-\mathrm{x}_{1}=\mathrm{x}_{2}=\mathrm{y}_{2}$.

O próximo passo acarretará na contradição que queremos. Por esta última igualdade, e das hipóteses da proposição, tem-se:

$$
\mathrm{Q}_{2}=\mathrm{x}_{2}+\mathrm{y}_{2}=2 \mathrm{av} \geq 2 \mathrm{v} \geq 2(\mathrm{u}+1)=2 \mathrm{u}+2 \geq 2 \mathrm{~N}+2>2 \mathrm{~N}+1 \geq \mathrm{T} .
$$


Ou seja,

$$
\mathrm{Q}_{2}>\mathrm{T},
$$

uma contradição.

Caso 2 Por outro lado, suponha agora que a $<0$ na mesma equação $\mathrm{x}_{2}-\mathrm{x}_{1}=\mathrm{av}$. Iremos chegar, por caminhos ligeiramente semelhantes, na contradição $Q_{1}>T$.

De (1), ainda temos

$$
\begin{gathered}
\mathrm{v}\left(\mathrm{y}_{1}-\mathrm{y}_{2}\right)=\mathrm{uav} \\
\mathrm{y}_{1}-\mathrm{y}_{2}=\mathrm{ua} .
\end{gathered}
$$

Considere $b=-a>0$. Desta forma,

$$
\mathrm{x}_{1}-\mathrm{x}_{2}=\mathrm{bv}
$$

e

$$
\mathrm{y}_{2}-\mathrm{y}_{1}=\mathrm{ub}
$$

De $\mathrm{b} \geq 1$ e somando $\mathrm{x}_{1}-\mathrm{x}_{2}=\mathrm{bv}$ com $\mathrm{y}_{2}-\mathrm{y}_{1}=\mathrm{ub}$, temos

$$
\left(\mathrm{x}_{1}-\mathrm{x}_{2}\right)+\left(\mathrm{y}_{2}-\mathrm{y}_{1}\right)=\mathrm{bv}+\mathrm{ub} \geq \mathrm{v}+\mathrm{u} \geq \mathrm{v}+\mathrm{N}>\mathrm{N}+\mathrm{N}=2 \mathrm{~N},
$$

de onde decorre que

$$
\mathrm{x}_{1}-\mathrm{x}_{2}+\mathrm{y}_{2}-\mathrm{y}_{1} \geq 2 \mathrm{~N}+1 \text {. }
$$

Lembremos que $\mathrm{T}=2 \mathrm{~N}+1$ ou $\mathrm{T}=2 \mathrm{~N}$, de modo que $\mathrm{T} \leq 2 \mathrm{~N}+1$.

Então, como por hipótese $\mathrm{x}_{1}-\mathrm{y}_{1} \leq 0$, temos:

$$
\mathrm{x}_{1}-\mathrm{x}_{2}+\mathrm{y}_{2}-\mathrm{y}_{1} \leq \mathrm{y}_{2}-\mathrm{x}_{2} \leq \mathrm{y}_{2} \leq \mathrm{T} \leq 2 \mathrm{~N}+1
$$

Logo, de (5) e de (6),

$$
\mathrm{x}_{1}-\mathrm{x}_{2}+\mathrm{y}_{2}-\mathrm{y}_{1}=2 \mathrm{~N}+1
$$

e as desigualdades em (6) na verdade são igualdades. Assim, $\mathrm{T}=2 \mathrm{~N}+1$.

As igualdades em (6) ainda implicam que $\mathrm{y}_{2}-\mathrm{x}_{2}=\mathrm{y}_{2}=2 \mathrm{~N}+1$, o que só é possível se $\mathrm{x}_{2}=0$.

Desta forma, de $(7), \mathrm{x}_{1}-0+2 \mathrm{~N}+1-\mathrm{y}_{1}=2 \mathrm{~N}+1$, e daí $\mathrm{x}_{1}=\mathrm{y}_{1}$.

Logo, bv $=\mathrm{x}_{1}-\mathrm{x}_{2}=\mathrm{x}_{1}=\mathrm{y}_{1}$.

O próximo passo acarretará na contradição que queremos. Por esta última igualdade, e das hipóteses da proposição, tem-se:

$$
\mathrm{Q}_{1}=\mathrm{x}_{1}+\mathrm{y}_{1}=2 \mathrm{bv} \geq 2 \mathrm{v} \geq 2(\mathrm{u}+1)=2 \mathrm{u}+2 \geq 2 \mathrm{~N}+2>2 \mathrm{~N}+1 \geq \mathrm{T},
$$

ou seja, $\mathrm{Q}_{1}>\mathrm{T}$, uma nova contradição,

o que conclui, enfim a prova da unicidade de $\mathrm{S}$ para todos os casos possíveis para o inteiro não nulo a. 


\section{Como adivinhar $\mathrm{x}$ e $\mathrm{y}$}

Bem, até aqui vimos como escolher os multiplicadores u e v e também provamos que não há repetição de somas $\mathrm{S}$ com essas escolhas. Como descobrir, porém, as quantidades x e y a partir da informação do valor de S? Certamente não será memorizando todas as possíveis somas $\mathrm{S}$ e os respectivos valores de x e y; afinal, T poderá ser qualquer natural!

Ora, como $\mathrm{S}=\mathrm{ux}+\mathrm{vy}$, tomando $\mathrm{v}-\mathrm{u}=\mathrm{m}$, temos:

$$
S=x u+y(u+m)=x u+y u+y m
$$

então, somando xm dos dois lados:

$$
\begin{gathered}
\mathrm{S}+\mathrm{xm}=\mathrm{xu}+\mathrm{yu}+\mathrm{ym}+\mathrm{xm}= \\
\mathrm{u}(\mathrm{x}+\mathrm{y})+\mathrm{m}(\mathrm{x}+\mathrm{y})= \\
(\mathrm{u}+\mathrm{m})(\mathrm{x}+\mathrm{y})= \\
\mathrm{v}(\mathrm{x}+\mathrm{y}) \\
\mathrm{S}+\mathrm{xm}=\mathrm{v}(\mathrm{x}+\mathrm{y}) .
\end{gathered}
$$

Logo, x será aquele valor que, multiplicado por m e somado a $\mathrm{S}$, resulta em um múltiplo de v , o que teoricamente enseja a descoberta de $\mathrm{x}$.

Analogamente, se subtrairmos ym dos dois lados na fórmula de S:

$$
S-y m=x u+y(u+m)-y m=u(x+y) .
$$

Assim, y será aquele valor que, multiplicado por m e subtraído de S, resulta em um múltiplo de u. Não parece ser simples encontrar $\mathrm{x}$ ou y por estes dois critérios. No entanto, se $\mathrm{u}$ e $\mathrm{v}$ forem consecutivos positivos (logo coprimos), então $\mathrm{m}=1$ e daí y será o número que subtraído de $\mathrm{S}$ resulta em múltiplo de $\mathrm{u}$, o que torna as operações mais fáceis.

\section{Um exemplo}

Enfim, analisemos uma situação concreta por meio de um diálogo, com T = 200, entre o adivinhador e participante. Os multiplicadores escolhidos serão os números inteiros consecutivos e coprimos $\mathrm{u}=100 \mathrm{e} \mathrm{v}=101$, maiores ou iguais à metade de 200, como manda a proposição; sendo assim, $\mathrm{m}=1$ e y será o número que subtraído de $\mathrm{S}$ resulta em um múltiplo de 100:

Adivinhador: Vamos jogar? Escolha uma quantidade de bolinhas entre 0 e 200.

Participante: Escolhida.

Adivinhador: Distribua nas duas mãos. Multiplique a menor quantidade por 100 e a maior por 101.

Participante: Feito.

Adivinhador: Some os resultados. Quanto deu? 


\section{Participante: Deu 9.580 .}

Adivinhador: Então, das 200 bolinhas, você escolheu 95, sendo 15 em uma mão e 80 na outra. Participante: Como você descobriu?

Adivinhador: Vamos lá: $\mathrm{S}=9.580$. Então, y é o valor que, subtraído de 9.580, resulta num múltiplo de 100. Como $9.580-80=9.500$, então y $=80$ é a maior quantidade que você escolheu distribuir numa das mãos.

Logo, isolando $\mathrm{x}$ em $\mathrm{S}=9.580=100 \mathrm{x}+101 \cdot 80$, tenho que

$$
\mathrm{x}=\frac{9.580-101 \cdot 80}{100}=15 .
$$

Esta é a menor quantidade escolhida. Você escolheu, portanto, um total de 95 bolinhas.

Ainda pode ficar uma dúvida. Caso pegássemos outro valor para y que subtraído de 9.580 também resultasse em um múltiplo de 100, por acaso não teríamos uma solução diferente, contrariando a proposição que ora provamos? A unicidade mostra ser isto impossível, porém, vejamos o que ocorreria nesse exemplo, a título de ilustração.

Se $y=180$, portanto, teríamos $9.580-180=9.400$, também um múltiplo de 100 . O problema ocorre agora, ao tentarmos encontrar $\mathrm{x}$ :

$$
\mathrm{x}=\frac{9.580-101 \cdot 180}{100}=-86
$$

Isto é, x seria negativo, o que não condiz com a mágica e tampouco com as hipóteses de nossa proposição.

\section{Considerações no ensino}

Naturalmente, a demonstração algébrica da validade do resultado é um tanto quanto complexa para ser mostrada a alunos de Ensino Médio, a menos que seja feita em alguma atividade extraclasse. No entanto, de posse do resultado, vale a aplicação da mágica aqui apresentada por despertar no estudante o espírito de investigação e questionamento do porquê de ela funcionar, servindo, quem sabe, como motivação para que o próprio aluno busque uma prova, com auxílio do seu professor. 


\section{Referências}

[1] Santos, R. C. dos \& Gontijo, C. H. "Uma mágica desafiadora". Revista do professor de Matemática. São Paulo: SBM, no 96. pp.31-32, 2018.

[2] Shokranian, S S; Soares, M. \& Godinho, H. Teoria dos Números. Brasília, UnB, 1999.

Rogério César dos Santos Faculdade UnB - Planaltina <rogerc@unb.br>

Paulo Eduardo de Brito Faculdade UnB - Planaltina <pedebrito@unb.br>

Wescley Well Vicente Bezerra

Faculdade UnB - Planaltina <wescley@unb.br>

Carlos Derli Almeida Cornélio Faculdade UEG - Formosa - Go <matcarlosderli@gmail.com>

Recebido: 02/03/2020

Publicado: 08/05/2020 